\title{
Urgent Procedure
}

National Cancer Institute

\section{Source}

National Cancer Institute. Urgent Procedure. NCI Thesaurus. Code C100031.

Deferring an urgent procedure increases risk of adverse outcome or clinical

deterioration. Clinical situation warrants performing urgent procedures during the current episode of care to avoid compromising patient safety. (ACC) 